\title{
Pregnancy outcomes following pelvic surgery during pregnancy: a nationwide population-based study in Korea
}

\author{
Hyun-Woong Cho ${ }^{1}$, Geum Joon Cho ${ }^{1}$, Jin Hwa Hong ${ }^{1}$, and Jae Kwan Lee ${ }^{1}$ \\ ${ }^{1}$ Korea University Guro Hospital
}

July 22, 2020

\begin{abstract}
Objective To investigate the risk of adverse birth outcomes for women who underwent non-obstetric abdominal surgery during pregnancy compared with that of those who did not undergo surgery. Design Retrospective cohort study Setting Korea National Health Insurance (KNHI) claims database Population A total of 8,167 women who did non-obstetric surgery and 3,710,123 women who did not undergo surgery and in 2006-2016. Methods The two groups were compared using a multivariate Cox proportional hazard model. The primary outcome assessed was neonatal complications and secondary outcomes were obstetric complications. Main Results Multivariate Cox proportional hazards regression revealed that risk of preterm birth [hazard ratio (HR) 2.06, 95\% confidence interval (CI) 1.86-2.29], low birth weight (LBW) (HR 1.65, 95\% CI 1.49-1.82), C/S (HR 1.14, 95\% CI 1.09-1.19), gestational hypertension (HR 1.36, 95\% CI 1.19-1.56), and placenta previa (HR 1.57, 95\% CI 1.33-1.87) was significantly higher in women who underwent non-obstetric surgery during pregnancy compared to women who did not undergo such surgery. When comparing the laparoscopic and laparotomy groups for risk of fetal outcomes, the risk of LBW was significantly decreased in laparoscopic adnexal resection during pregnancy compared to that of laparotomy (HR $0.62,95 \%$ CI 0.40-0.95). Conclusion: Non-obstetric pelvic surgery during pregnancy was associated with higher risk of preterm birth, LBW, gestational hypertension, placenta previa, placental abruption, and C/S. Although the benefits and safety of laparoscopy during pregnancy appear similar to those of laparotomy in pregnancy outcomes, laparoscopic adnexal mass resection was associated with a lower risk of LBW.
\end{abstract}

\section{Introduction}

One to two percent of women undergo non-obstetric surgery during pregnancy, including appendectomy, gynecologic surgery, and cancer surgery. ${ }^{1}$ Non-obstetric surgery during pregnancy is associated with adverse obstetric and fetal outcomes. In a Canadian study of 2,565 pregnant women, there was increased risk of spontaneous abortion after general anesthesia. ${ }^{2}$ A Swedish registry study showed increased risk of preterm birth and growth restriction in the surgery group. ${ }^{3}$ In a 6.5 million patient cohort in the UK, non-obstetric surgery during pregnancy was associated with adverse birth outcomes such as stillbirth, preterm delivery, low birth weight, and cesarean section. ${ }^{4}$ The American College of Obstetricians and Gynecologists (ACOG) committee noted that, although no teratogenic effect of anesthetics during pregnancy has been proven, obstetric and fetal complications may increase.

Laparoscopic surgery during pregnancy has become widely used, although there has been concern for uterine injury from trocar placement and fetal malperfusion due to pneumoperitoneum during laparoscopic surgery over the past two decades. ${ }^{5}$ Several systematic reviews and meta-analyses have suggested that laparoscopic surgery may be similar or preferable to laparotomy during pregnancy. ${ }^{6-11}$ The majority of previous studies, however, has included a small number of patients and targeted only certain operations, such as appendectomy or ovarian surgery. Recently, a Japanese registry study confirmed the advantages of laparoscopic surgery for benign diseases compared with laparotomy in 6,018 pregnant women who underwent surgery. However, that study was limited because it did not evaluate risk of major obstetric/fetal complications, including gestational 
hypertension, cesarean section, and low birth weight, but only assessed abortion, stillbirth within 7 days after surgery, and premature birth during hospitalization. ${ }^{12}$

The aim of this study was to investigate the risk of adverse pregnancy outcomes for women who underwent non-obstetric abdominal surgery during pregnancy compared with those who did not undergo such surgery. The study also compared maternal and fetal outcomes after laparoscopy versus laparotomy for benign disease during pregnancy.

\section{Materials and methods}

\section{Healthcare system in Korea}

Since 2000, various health insurance systems in South Korea have merged into a single system run by the National Health Insurance Service (NHIS). Consequently, most people living in South Korea are currently insured by the NHIS. Our study data were collected from the Korea National Health Insurance (KNHI) claims database from 2007-2015. In Korea, $97 \%$ of the population is obligated to enroll in the KNHI program; the remaining 3\% are under the Medical Aid Program. Therefore, the KNHI claims database contains information on all claims for approximately 50 million Koreans, and almost all information about disease incidence can be obtained from this centralized database, with the exception of procedures not covered by insurance, such as cosmetic surgery.

\section{Study population}

A flowchart of patient enrollment is shown in Fig. 1. Using the KNHI claims database, we identified all women who gave birth from January 2007 to December 2015. Inclusion criteria were as follows: women who gave birth between January 2006 and December 2016, and had undergone the National Health Screening Program for Infant and Children (NHSP-IC) visit to evaluate neonatal characteristics. Women with no or missing NHSP-IC data were excluded from this study. This study was approved by the Institutional Review Board of Korea University Medical Center (2020GR0109).

We identified pregnant women who underwent non-obstetric surgery from the database. Patients who underwent non-obstetric surgery at the time of cesarean delivery were excluded. In this study, non-obstetric surgeries (by laparoscopy or laparotomy) included the two most common operative procedures for benign diseases: appendectomy and adnexal mass resection. The operative procedures were identified by the presence of a Korea Medical Insurance electronic data interchange (EDI) code (Appendectomy: Q2861/Q2862/Q2863, Adnexal mass resection: R4430/R4421). Laparoscopic surgery was identified by the associated Korea Medical Insurance EDI code (N0031001).

\section{Outcomes}

The primary pregnancy outcome was neonatal complications, including premature birth and low birth weight (LBW). Secondary outcomes were obstetric complications, including gestational hypertension, gestational diabetes mellitus (GDM), postpartum hemorrhage ( $\mathrm{PPH})$, placental abruption, placenta previa, cesarean section $(\mathrm{C} / \mathrm{S})$, and length of hospital stay after surgery. Patient characteristics such as maternal age and parity were evaluated using the KNHI claims database. The length of hospital stay after surgery and the time interval between surgery and delivery were also measured.

\section{Statistical analysis}

Continuous and categorical variables were expressed as mean \pm SD and percentages, respectively. Clinical characteristics were compared using the $t$-test for continuous variables and the $\chi^{2}$ test for categorical variables. Multivariate regression analysis models were used to estimate the adjusted hazard ratios (HRs) and $95 \%$ confidence intervals (CIs) for primary and secondary endpoints. All tests were two-tailed, and pvalues $<0.05$ were considered statistically significant. Statistical analyses were performed using SPSS version 18 (IBM Corp., Armonk, NY, USA).

\section{Results}


From a total of 3,276,776 of women who gave birth during 2007-2015, 8,167 (0.25\%) underwent non-obstetric abdominal surgery, including 5,109 laparotomy patients and 3,108 laparoscopy patients. Table 1 shows the pregnancy characteristics of patients with or without surgery during pregnancy.

Table 1 shows the general and pregnancy characteristics of patients between pregnancies with and without surgery. For pregnancies in the surgery group, maternal age $<35$ years, nulliparity, preterm birth, LBW, gestational hypertension, GDM, C/S, placenta previa, and placenta abruption were more common than in pregnancies in the group that did not undergo surgery. Table 2 shows the general and pregnancy characteristics of the laparoscopy and laparotomy groups. When comparing laparoscopy and laparotomy, in the laparoscopy group, maternal age $<35$ years, nulliparity, C/S, gestational hypertension, GDM, and placenta previa were more common than in the laparotomy group. Preterm delivery, low birth weight, and placenta abruption were less common in the laparoscopic group compared with the laparotomy group.

Figure 2 shows the results of Cox proportional hazards regression analyses with fetal and obstetric complications after controlling for age and parity. There was a statistically significant increase in risk of preterm birth (HR 2.06, 95\% CI 1.86-2.29), LBW (HR 1.65, 95\% CI 1.49-1.82), C/S (HR 1.14, 95\% CI 1.09-1.19), gestational hypertension (HR 1.36, 95\% CI 1.19-1.56), and placenta previa (HR 1.57, 95\% CI 1.33-1.87) in both the laparotomy and laparoscopy groups. The risk of placental abruption was significantly increased only in the laparotomy group (HR 1.59, 95\% CI 1.12-2.27).

When comparing the laparoscopic and laparotomy groups for risk of fetal outcomes, the risk of LBW was significantly decreased in laparoscopic adnexal resection during pregnancy compared to laparotomy (HR $0.62,95 \%$ CI 0.40-0.95) (Table 3). Otherwise, there were no statistically significant differences between the two groups. In addition, in the laparoscopic group, the hospital stay was statistically significantly shorter than for the laparotomy group $(6.13 \pm 2.76$ vs. $5.62 \pm 2.75$ days, $\mathrm{P}<0.001)$.

\section{Discussion}

\section{Main finding}

This study showed that there was higher incidence and risk of preterm birth, low birth weight, gestational hypertension, placenta previa, placenta abruption, and cesarean section in women who underwent nonobstetric abdominal surgery during pregnancy compared to pregnant women who did not undergo such surgery. However, when the laparoscopic surgery performed more than 6 months before delivery, there was no increased risk of obstetric and fetal complications including premature birth and LBW. Although there was no significant difference in most obstetric and fetal complications between laparotomy and laparoscopy group, the risk of LBW was significantly increased in the laparotomy group compared with laparoscopy group among the pregnant women who underwent ovarian resection.

\section{Strengths and limitations}

Our findings need to be interpreted in the context of some inherent limitations of a claims database. First, this was a retrospective study. Second, other abdominopelvic surgeries such as cholecystectomy and myomectomy were excluded from the analysis due to their small number, indicating the need for additional research on these procedures. Third, there was a lack of clinical information about gestational age. Therefore, we estimated the time interval between surgery and delivery to control bias due to time of surgery. Fourth, because the study only included data on patients who experienced live birth, no information about stillbirth was available. However, it is possible that the effect of surgery on the fetus was fully evaluated by measuring the incidence of low birth weight and preterm birth. Finally, some important individual data about lifestyle behaviors, such as smoking and alcohol drinking habits, body mass index, and severity of comorbid conditions, were not available.

Nevertheless, there were many strengths to this study and the research approach. This is the first nationwide study to report the risk of adverse obstetric and fetal outcomes following non-obstetric pelvic surgery during pregnancy in Korea. This is also the largest such study in Asia, the second largest study worldwide, and is based on a registry database from 3.2 million pregnancies. Furthermore, it is the first large-scale study to 
analyze the risk of various obstetric and fetal complications according to laparoscopic/open surgery, type of surgery, and time of surgery during pregnancy.

\section{Interpretation}

This study demonstrated higher risk of preterm birth, low birth weight, gestational hypertension, placenta previa, placenta abruption, and cesarean section in women who underwent non-obstetric abdominal surgery during pregnancy compared to pregnant women who did not. This result is consistent with previous large studies. ${ }^{2-4,13,14}$ A Swedish study of 2 million pregnancies suggested increased risk for fetuses of pregnant women who underwent surgery to weigh $<2500 \mathrm{~g}$, to be delivered before 37 weeks, and to have an increased incidence of growth restriction compared with the total population. ${ }^{3}$ Although the risk associated with nonobstetric surgery is relatively low, surgical operations were associated with additional stillbirth (1 per 287 surgery), preterm delivery (1 per 31 surgery), LBW (1 per 39 surgery), and C/S (1 per 25 surgery) according to a cohort study of 6.5 million pregnancies in England. ${ }^{4}$ In a Taiwanese registry-based study of 150,000 pregnancies, non-obstetric surgery during pregnancy was associated with higher risk of spontaneous abortion ( $4.23 \%$ vs. $2.43 \%$, adjusted odd ratio [aOR]:1.53; $95 \%$ CI: $1.01-2.31$ ), pre-eclampsia/eclampsia (2.60\% vs. 1.01\%, aOR: $2.35 ; 95 \%$ CI: $1.30-4.23)$, gestational diabetes (2.38\% vs. $0.69 \%$, aOR: $3.12 ; 95 \%$ CI: $1.69-5.78)$, prematurity (9.06 vs. $4.90 \%$, aOR: $3.31 ; 95 \%$ CI: $2.54-4.31$ ), and cesarean section ( $43.55 \%$ vs. $33.76 \%$, aOR: 1.41; $95 \%$ CI: $1.17-1.71){ }^{13}$

In this study, although the laparoscopic group tended to have a lower risk of fetal complications than the laparotomy group, there was no significant difference between the groups. However, in the pregnant women who underwent ovarian resection, the risk of LBW was significantly increased in the laparotomy group. The effect and safety of laparoscopic surgery on pregnancy have been discussed for many years. In 1997, a Swedish health registry study suggested that there was no difference in birth weight, gestational duration, growth restriction, infant survival, or fetal malformations for patients undergoing laparoscopy versus laparotomy in singleton pregnancies between 4 and 20 weeks of gestation. A recent Japanese registry study showed that laparoscopic surgery had advantages in short-term fetal adverse events, incidence of blood transfusion, operative time, and hospital stay. ${ }^{12}$ However, there are some limitations to these studies. Since the Swedish study was conducted 20 years ago, the results of the study were limited for evaluating the impact of laparoscopic surgery on pregnancy. In addition, there were very few laparoscopic surgeries during the second or third trimester. The Japanese study was a recent study that used a large national database, which had an advantage of performing propensity matching to correct for possible confounders such as age, BMI, gestational age at surgery, smoking, operative procedure, emergent maternal transport, emergent surgery within 2 days of admission, and the average ratio of laparoscopies to laparotomies performed at each hospital. However, LBW, which is a major complication of surgery during pregnancy, and preterm birth were not identified in the Japanese study. Moreover, the risk of multiple obstetric complications such as gestational hypertension, gestational diabetes, and $\mathrm{C} / \mathrm{S}$ that have been associated with surgery during pregnancy was not assessed. On the other hand, in our study, the overall incidence of preterm birth and LBW was evaluated using the National Health Screening Program for Infants and Children (NHSP-IC). Obstetric complications such as gestational hypertension, GDM, PPH, placental abruption, placenta previa, C/S, and length of hospital stay after surgery were also evaluated in this study.

There was a significant difference in risk of fetal complications according to the time of surgery during pregnancy. Comparison between the laparoscopy and open groups indicated that there was no significant difference in fetal and obstetric complications, although there was a tendency for increased risk of preterm birth and LBW. However, there was no increased risk of obstetric and fetal complications, including premature birth and LBW, in women who underwent laparoscopic surgery more than 6 months before delivery. This is consistent with the results of previous studies. In a systematic review of 12,452 pregnancies, surgery in the first trimester did not appear to increase major birth defects. ${ }^{15}$ In a Taiwanese registry study, deliveries that were associated with non-obstetric surgery in the third trimester had a 3.79-fold (95\% CI, 1.20-11.96) increase in OR compared to those with surgery in the first trimester. ${ }^{13}$

This study showed that hospital stay in the laparoscopy group was statistically significantly shorter than 
that in the laparotomy group. This is one of the great advantages of laparoscopic surgery and has also been reported in several studies. According to the Society of American Gastrointestinal and Endoscopic Surgeons (SAGES) guidelines, benefits of laparoscopy during pregnancy appear similar to those in nonpregnant patients including less postoperative pain, less postoperative ileus, decreased length of hospital stays, and faster return to work. ${ }^{5}$ A Japanese registry study also showed that the laparoscopy group had a significantly shorter hospital stay ( 9.2 vs. 5.9 days, $\mathrm{p}<.001$ ) compared with the laparotomy group. ${ }^{12}$

\section{Conclusion}

Non-obstetric pelvic surgery during pregnancy was associated with higher risk of preterm birth, LBW, gestational hypertension, placenta previa, placental abruption, and C/S. Although the benefits and safety of laparoscopy during pregnancy appear similar to those of laparotomy in pregnancy outcomes, laparoscopic adnexal mass resection was associated with a lower risk of LBW. The results of this study should be considered when selecting surgical and procedural approaches to optimize patient outcomes.

\section{Disclosure of interests}

None declared. Completed disclosure of interest forms are available to view online as supporting information.

\section{Contribution to authorship}

HWC: Study concept, analysis and interpretation of the data, drafting of manuscript, manuscript revision, approval of final version. GJC, JKL: analysis and interpretation of the data, manuscript revision, approval of final version. JHH: Manuscript revision, interpretation and presentation of the data.

\section{Funding}

No funding to declare.

\section{Details of ethics approval}

All procedures were in accordance with the ethical standards of the Institutional Review Board of Korea University Medical Center (2020GR0109) and with the 1964 Declaration of Helsinki and its later amendments or comparable ethical standards. Informed patient consent was not required. Retrospective data collection was anonymized and routinely collected as part of clinical practice and used for audit purposes.

\section{Acknowledgments}

We thank Korea National Health Insurance for their cooperation in providing access to the claims database.

\section{References}

1 Rasmussen AS, Christiansen CF, Uldbjerg N, Nørgaard M. Obstetric and non-obstetric surgery during pregnancy: A 20-year Danish population-based prevalence study. BMJ Open 2019;9:e028136.

2 Duncan PG, Pope WD, Cohen MM, Greer N. Fetal risk of anesthesia and surgery during pregnancy. Anesthesiology 1986;64:790-4.

3 Reedy MB, Källén B, Kuehl TJ. Laparoscopy during pregnancy: a study of five fetal outcome parameters with use of the Swedish Health Registry. Am J Obstet Gynecol 1997;177:673-9.

4 Balinskaite V, Bottle A, Sodhi V, Rivers A, Bennett PR, Brett SJ, et al. The Risk of Adverse Pregnancy Outcomes Following Nonobstetric Surgery During Pregnancy: Estimates From a Retrospective Cohort Study of 6.5 Million Pregnancies. Ann Surg 2017;266:260-6.

5 Pearl JP, Price RR, Tonkin AE, Richardson WS, Stefanidis D. SAGES guidelines for the use of laparoscopy during pregnancy. Surg Endosc 2017;31:3767-82.

6 Bunyavejchevin S, Phupong V. Laparoscopic surgery for presumed benign ovarian tumor during pregnancy. Cochrane Database Syst Rev 2013:CD005459. 
7 Liu YX, Zhang Y, Huang JF, Wang L. Meta-analysis comparing the safety of laparoscopic and open surgical approaches for suspected adnexal mass during the second trimester. Int J Gynaecol Obstet2017;136:272-9.

8 Sedaghat N, Cao AM, Eslick GD, Cox MR. Laparoscopic versus open cholecystectomy in pregnancy: a systematic review and meta-analysis. Surg Endosc 2017;31:673-9.

9 Walker HG, Al Samaraee A, Mills SJ, Kalbassi MR. Laparoscopic appendicectomy in pregnancy: a systematic review of the published evidence. Int J Surg 2014;12:1235-41.

10 Webb KE, Sakhel K, Chauhan SP, Abuhamad AZ. Adnexal mass during pregnancy: a review. Am $J$ Perinatol 2015;32:1010-6.

11 Wilasrusmee C, Sukrat B, McEvoy M, Attia J, Thakkinstian A. Systematic review and meta-analysis of safety of laparoscopic versus open appendicectomy for suspected appendicitis in pregnancy. $\mathrm{Br} J$ Surg 2012;99:1470-8.

12 Shigemi D, Aso S, Matsui H, Fushimi K, Yasunaga H. Safety of Laparoscopic Surgery for Benign Diseases during Pregnancy: A Nationwide Retrospective Cohort Study. J Minim Invasive Gynecol2019;26:501-6.

$13 \mathrm{Yu}$ CH, Weng SF, Ho CH, Chen YC, Chen JY, Chang YJ, et al. Pregnancy outcomes following nonobstetric surgery during gestation: a nationwide population-based case-control study in Taiwan. BMC Pregnancy Childbirth 2018;18:460.

14 Mazze RI, Källén B. Reproductive outcome after anesthesia and operation during pregnancy: a registry study of 5405 cases. Am J Obstet Gynecol 1989;161:1178-85.

15 Cohen-Kerem R, Railton C, Oren D, Lishner M, Koren G. Pregnancy outcome following non-obstetric surgical intervention. Am J Surg 2005;190:467-73.

\section{Hosted file}

Tables.docx available at https://authorea.com/users/345145/articles/471468-pregnancyoutcomes-following-pelvic-surgery-during-pregnancy-a-nationwide-population-based-studyin-korea

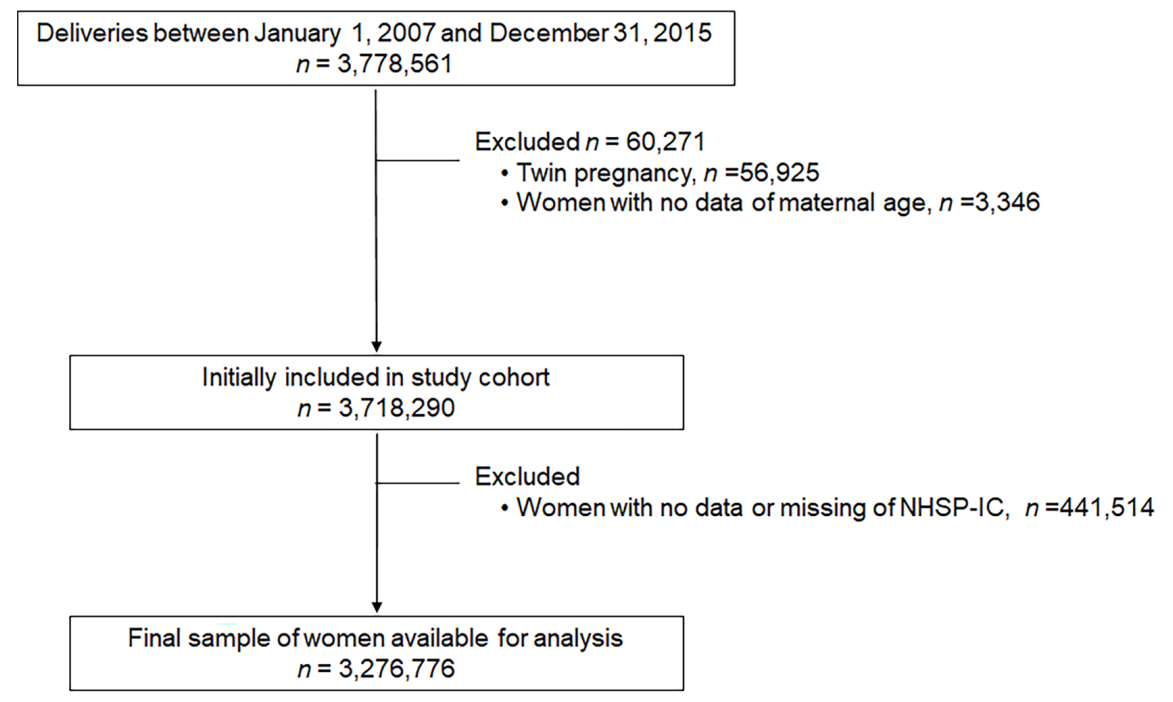


Preterm birth Overall 2.06(1.86-2.29) laparotomy 2.19(1.93-2.49) laparoscopy $1.85(1.55-2.21)$

LBW

Overall 1.65(1.49-1.82) laparotomy $1.68(1.48-1.91)$ laparoscopy $1.59(1.35-1.87)$

$\mathbf{C} / \mathbf{S}$

Overall 1.14(1.09-1.19) laparotomy 1.12(1.06-1.19) laparoscopy $1.17(1.09-1.26)$

Gestational hypertension Overall 1.36(1.19-1.56) laparotomy $1.36(1.15-1.61)$ laparoscopy $1.36(1.10-1.69)$

\section{GDM}

Overall 1.04(0.93-1.16) laparotomy $1.00(0.86-1.15)$ laparoscopy 1.11(0.94-1.32)

Placenta previa Overall 1.57(1.33-1.87) laparotomy $1.53(1.23-1.90)$ laparoscopy $1.65(1.27-2.16)$

Placental abruption Overall 1.43(1.06-1.91) laparotomy $1.59(1.12-2.27)$ laparoscopy $1.16(0.69-1.97)$

\section{PPH}

Overall 1.06(0.98-1.14) laparotomy $1.08(0.97-1.19)$ laparoscopy 1.02(0.89-1.16)

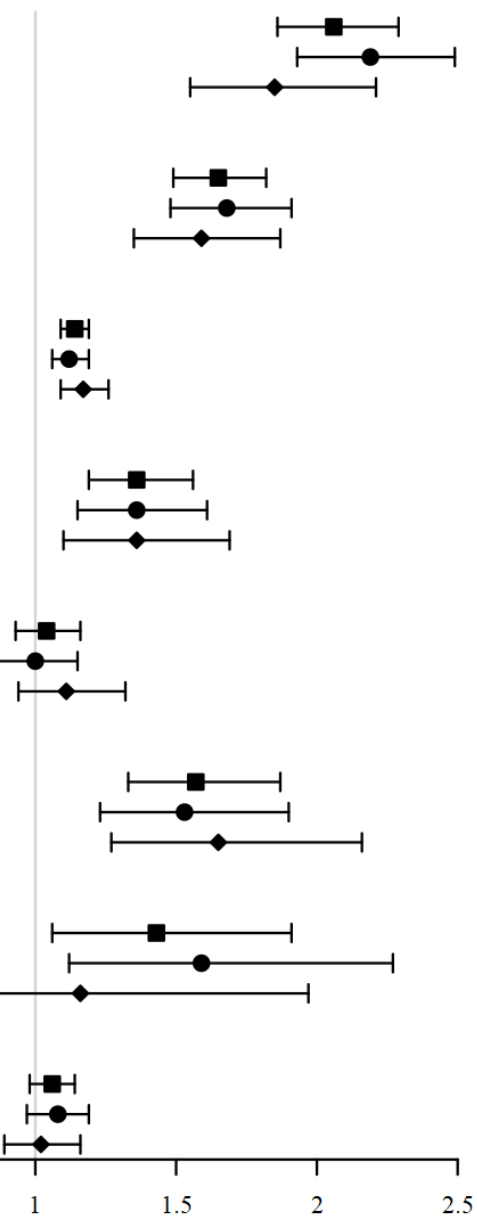

